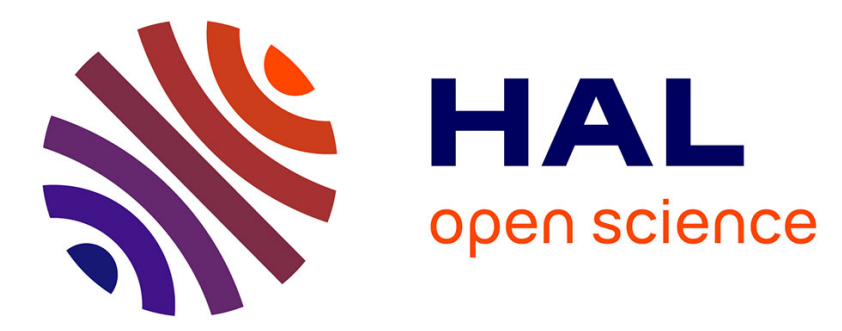

\title{
Complémentarité intertemporelle de la production, coordination de l'activité économique et croissance hors de l'équilibre
}

Jean-Luc Gaffard, Mario Amendola

\section{- To cite this version:}

Jean-Luc Gaffard, Mario Amendola. Complémentarité intertemporelle de la production, coordination de l'activité économique et croissance hors de l'équilibre. Revue Economique, 1995, 46 (6), pp.15271540. 10.3406/reco.1995.409750 . hal-01026557

\section{HAL Id: hal-01026557 \\ https://hal-sciencespo.archives-ouvertes.fr/hal-01026557}

Submitted on 21 Jul 2014

HAL is a multi-disciplinary open access archive for the deposit and dissemination of scientific research documents, whether they are published or not. The documents may come from teaching and research institutions in France or abroad, or from public or private research centers.
L'archive ouverte pluridisciplinaire HAL, est destinée au dépôt et à la diffusion de documents scientifiques de niveau recherche, publiés ou non, émanant des établissements d'enseignement et de recherche français ou étrangers, des laboratoires publics ou privés.

\section{(이)( $)$}

Distributed under a Creative Commons Attribution - NonCommercial - NoDerivatives 44.0 


\title{
Persée
}

http://www.persee.fr

\section{Complémentarité intertemporelle de la production, coordination de l'activité économique et croissance hors de l'équilibre.}

\author{
Jean-Luc Gaffard;Mario Amendola \\ Revue économique, Année 1995, Volume 46, Numéro 6 \\ p. $1527-1540$
}

Voir l'article en ligne

\section{Avertissement}

L'éditeur du site «PERSEE » - le Ministère de la jeunesse, de l'éducation nationale et de la recherche, Direction de l'enseignement supérieur, Sous-direction des bibliothèques et de la documentation - détient la propriété intellectuelle et les droits d'exploitation. A ce titre il est titulaire des droits d'auteur et du droit sui generis du producteur de bases de données sur ce site conformément à la loi n`98-536 du $1 \mathrm{er} \mathrm{juillet} 1998$ relative aux bases de données.

Les oeuvres reproduites sur le site «PERSEE » sont protégées par les dispositions générales du Code de la propriété intellectuelle.

Droits et devoirs des utilisateurs

Pour un usage strictement privé, la simple reproduction du contenu de ce site est libre.

Pour un usage scientifique ou pédagogique, à des fins de recherches, d'enseignement ou de communication excluant toute exploitation commerciale, la reproduction et la communication au public du contenu de ce site sont autorisées, sous réserve que celles-ci servent d'illustration, ne soient pas substantielles et ne soient pas expressément limitées (plans ou photographies). La mention Le Ministère de la jeunesse, de l'éducation nationale et de la recherche, Direction de l'enseignement supérieur, Sous-direction des bibliothèques et de la documentation sur chaque reproduction tirée du site est obligatoire ainsi que le nom de la revue et- lorsqu'ils sont indiqués - le nom de l'auteur et la référence du document reproduit.

Toute autre reproduction ou communication au public, intégrale ou substantielle du contenu de ce site, par quelque procédé que ce soit, de l'éditeur original de l'oeuvre, de l'auteur et de ses ayants droit.

La reproduction et l'exploitation des photographies et des plans, y compris à des fins commerciales, doivent être autorisés par l'éditeur du site, Le Ministère de la jeunesse, de l'éducation nationale et de la recherche, Direction de l'enseignement supérieur, Sous-direction des bibliothèques et de la documentation (voir http://www.sup.adc.education.fr/bib/ ). La source et les crédits devront toujours être mentionnés. 


\title{
Complémentarité intertemporelle de la production, coordination de l'activité économique et croissance hors de l'équilibre
}

\author{
Mario Amendola* \\ Jean-Luc Gaffard **
}

\begin{abstract}
Cet article est destiné à montrer quelques aspects importants de processus de changement hors de l'équilibre ; en particulier la distorsion de la capacité productive associée a un changement structurel et ses effets au cours du temps. II est mis en évidence, à titre d'exemple, qu'un changement du taux de prélèvement n'est pas une condition suffisante pour obtenir un taux de croissance plus élevé de l'économie.
\end{abstract}

Classification JEL : C62, C63, E 32, 041.

\section{PROBLÈMES ET MÉTHODES DE DYNAMIQUE ÉCONOMIQUE}

L'analyse dynamique en économie devrait consister à traiter de problèmes par essence dynamiques, plutôt qu'à se référer à des méthodes supposées dynamiques. Mais qu'est-ce qu'un problème de dynamique en économie ? Tout dépend de ce que l'on entend par changement. Chacun s'accorde pour dire que c'est la considération du changement qui est ce qui distingue la dynamique de la statique. Dans le cas des analyses de croissances régulières, le changement a une signification purement quantitative puisqu'il consiste dans l'augmentation du niveau d'activité d'un système prođuctif dont les caractéristiques structurelles et les modes de fonctionnement sont établis une fois pour toutes. Dans le cas des analyses de croissances cycliques basées essentiellement sur l'introduction de non-linéarités, celle-ci est seulement destinée à rendre possible la définition de différentes configurations du sentier d'équilibre suivi par l'économie. Ce sont là des interprétations réductrices du changement et sûrement pas celles que nous avons ici à l'esprit, quand nous nous référons aux aspects dynamiques des phénomènes économiques.

La distinction effectuée par J.A. Schumpeter entre la croissance, explicitement définie comme un phénomène quantitatif, et le développement, défini

* University of Rome « La Sapienza ». Dipartimento di Scienze Economiche, via Andrea Cesalpino, Roma, Italie.

** University of Nice Sophia-Antipolis et CNRS-LATAPSES, 250, avenue AlbertEinstein, 06560 Valbonne, France. 
comme « un changement discontinu qui vient de l'intérieur du processus économique en raison de sa nature » (N. Georgescu-Roegen [1976], p. 245), aide à comprendre ce point. Elle permet, en effet, de souligner qu'un vrai problème de dynamique est en cause dès lors qu'il y a un changement qualitatif, à savoir un changement qui implique des modifications de structure et qui prend place suivant un processus irréversible. L'innovation qui implique la création de nouvelles ressources et la construction de nouveaux ensembles de choix est l'exemple emblématique de ce genre de changement. Mais un simple changement de rythme de la croissance ou un simple changement de technique sont de même nature. Dans tous ces cas, en effet, la structure productive existante est perturbée, son mode de fonctionnement est affecté de telle sorte qu'un problème de complémentarité intertemporelle surgit, qui requiert une coordination dans le temps de l'activité économique dans le but de rendre viable le processus engagé.

Dans cette perspective, le véritable enjeu d'une analyse économique dynamique est moins de considérer les propriétés d'un équilibre dans le temps que de comprendre à quelles conditions une économie, soumise à des perturbations qui la placent hors de l'équilibre, continue d'exister (reste viable). C'est à cette tâche qu'est dédié le présent article, quoique les réponses proposées ne puissent être que partielles et provisoires.

La question générale, qui vient d'être soulevée, requiert, en tout premier lieu, une mise en perspective de ce qu'il est convenu d'appeler la dynamique transitoire (section 2), pour ensuite pouvoir poser les bases d'une analyse spécifique des changements de régime de croissance qui mette l'accent sur le rôle des complémentarités intertemporelles (section 3). Un modèle sera alors proposé (section 4), qui permettra d'explorer, au moyen de simulations numériques, les conditions dans lesquelles une accélération de la croissance peut être obtenue à partir d'un déplacement de ressources de la consommation finale vers l'épargne et l'investissement (section 5).

\section{LA DYNAMIQUE TRANSITOIRE : LES LEÇONS DES DÉVELOPPEMENTS RÉCENTS, EN THÉORIE DE LA CROISSANCE}

L'accent mis sur les sources endogènes de croissance - que l'on se réfère à une augmentation du taux d'épargne, et par suite de la fraction du produit qui est investie, ou bien à un déplacement de ressources au bénéfice de la R\&D de manière à renforcer l'innovation - est la contribution distinctive des derniers développements en matière de théorie de la croissance. Cependant, l'apparition d'une capacité productive avec une structure différente, ou la production d'une nouvelle technologie ne renvoient pas simplement à une nouvelle relation entre facteurs et produit. L'affectation des ressources n'est que l'étape préliminaire d'un processus à travers lequel les changements prennent place. Or « the hard part is to model what happens then » (R. Solow, [1994], p.52). Et c'est exactement ce que les modèles de croissance endogène ne peuvent pas nous dire.

C'est un fait que les modèles de croissance endogène sont des modèles d'équilibre où le choix approprié des valeurs des paramètres clés, garanti par une prévision parfaite, fait que l'économie saute immédiatement au taux de croissance du régime régulier. En général, ces modèles n'exhibent pas de dyna- 
mique transitoire (cf. R. Barro, X. Sala, Y. Martin [1995]). Ils autorisent seulement une analyse comparative des différents sentiers de croissance endogène. Aucun des problèmes de complémentarité et/ou de coordination (qui surgissent, nécessairement, durant une phase de transition) ne peut être évoqué puisque les économies décrites sont toujours à l'équilibre. Les comparaisons effectuées sont néanmoins plus riches que celles qui procèdent de l'analyse standard, dans la mesure où il est désormais possible d'associer des sentiers de croissance différents à des valeurs différentes des paramètres de préférence, et de considérer les effets d'éventuelles politiques de croissance susceptibles d'agir sur ces valeurs.

Ainsi les modèles de croissance endogène, comme tous les modèles de croissance équilibrée, sont destinés à identifier les facteurs de croissance et à mesurer leurs contributions respectives à cette croissance, pour en déduire des propositions de politique économique. Mais il n'y a aucune tentative de comprendre le fonctionnement du mécanisme de croissance, exception faite, toutefois, de la référence, dans certains modèles, au rôle des rendements croissants.

Ce dernier point mérite qu'on s'y arrête pour souligner, d'abord, que l'existence de rendements croissants n'est pas strictement nécessaire pour obtenir une croissance endogène : le maintien d'une stricte complémentarité des facteurs suffit à y pourvoir (cf. E. Solow [1992], R. Barro, X. Sala, Y. Martin, ibid). Par ailleurs, ces rendements croissants, quand ils sont pris en considération, sont traités de telle manière à éviter de poser le problème d'une coordination éventuelle entre l'augmentation de l'offre et celle de la demande, qui est, pourtant, un problème typique de transition.

Dans un même ordre d'idées, la prétention des modèles de croissance endogène à faire place à une analyse normative - dans le sens où ils rendent possible de comparer différentes alternatives de politique économique et de sélectionner la meilleure (celle qui correspond au taux de croissance le plus élevé) - mérite, également, discussion. C'est un fait que, dans le contexte des théories de l'équilibre, les interventions de politique économique doivent être capables de mettre instantanément l'économie dans les conditions requises (lesquelles peuvent d'ailleurs être très restrictives), alors même que le déplacement de ressources, automatiquement identifié avec le résultat qu'il devrait engendrer, peut très bien, comme nous le verrons (section 5 ci-après), conduire l'économie à des situations de non-viabilité.

L'approche moderne en matière de théorie de la croissance, et d'ailleurs de théorie des cycles, consiste à éliminer toute référence à une dynamique transitoire. Les problèmes de coordination sont évacués comme ils l'étaient dans les modèles traditionnels. La présomption que la demande et l'offre se meuvent continuement en restant en ligne l'une avec l'autre est maintenue systématiquement. Elle repose sur une autre présomption qui est celle d'une synchronisation non moins systématique entre la consommation et l'investissement, c'est-à-dire de l'absence de difficultés liées à l'existence des complémentarités intertemporelles. Il n'est plus question comme dans les vieilles théories (celles de l'entredeux-guerres) de chercher à expliquer les mouvements de l'économie hors de l'équilibre, et de dissocier les positions d'équilibre des processus d'ajustement.

Or, précisément, vouloir prendre en considération la dynamique transitoire, c'est reconnaître l'existence de changements hors de l'équilibre et c'est donc reconnaître le poids des problèmes de complémentarité intertemporelle et de coordination. 


\section{L'ANALYSE HICKSIENNE DE LA TRAVERSE : ENJEUX ET LIMITES}

L'articulation dans le temps du processus de production est le caractère distinctif du modèle néo-autrichien. Dans ce modèle, la production apparaît comme un schéma pour transformer une séquence de facteurs primaires en une séquence de produit final. Le processus de production est verticalement intégré, ce qui rend possible à la fois de considérer, explicitement, une phase de construction et une phase d'utilisation de la capacité productive, et de souligner que la première vient avant la seconde. L'accent mis sur la structure temporelle du processus de production, et sur sa complémentarité intertemporelle, permet de positionner la transition entre deux techniques (une traverse) comme un véritable processus et d'analyser ce processus en le suivant dans son développement séquentiel (J.R. Hicks [1973]).

La complémentarité intertemporelle de la production est le principal lien inter-périodique dans une économie à un bien dont on analyse le passage d'une technique à une autre, sous l'hypothèse de plein rendement. Cette hypothèse implique, en effet, que tout le produit, qui n'est pas absorbé par la consommation des salariés engagés sur les processus de production existants et par la consommation à partir des profits, est utilisé pour faire démarrer de nouveaux processus. Le taux de natalité est, de cette façon, rendu endogène et détermine le sentier suivi par l'économie. Mais, surtout, un équilibre de flux est assuré à chaque période puisque l'offre est égale à la demande, et l'épargne est égale à l'investissement. Dans ces conditions, la structure de la capacité productive est progressivement transformée pour s'adapter à la nouvelle technique, à mesure que les ressources graduellement libérées sont investies dans cette dernière. Aucun problème de coordination ne surgit ; la coordination est assurée par le plein rendement.

Le pas en avant que représente l'analyse de la traverse est que, bien qu'il s'agisse essentiellement d'une approche d'équilibre, le fait de prendre en considération explicitement la structure temporelle de la production rend possible de traiter réellement de la dynamique transitoire de l'économie, c'est-à-dire d'éclairer « what happens on the way » (J.R. Hicks [1973], p. 10). De cela témoignent la démonstration de l'existence et la caractérisation de la cause de l'effet-machine de Ricardo. Un tel effet vient de la dissociation des facteurs et du produit, des coûts et des recettes au cours de la transition, dont le résultat est une chute temporaire du produit final et, donc, des ressources disponibles pour soutenir l'emploi, ce que seul l'accent mis sur la structure temporelle de la production permet de mettre en évidence.

Cependant, si l'analyse de la traverse, avec le modèle néo-autrichien de Hicks, permet d'identifier des effets de la complémentarité intertemporelle de la production, elle reste une analyse d'équilibre. Non seulement les problèmes de coordination sont évacués grâce à l'hypothèse de plein rendement, mais un sentier de croissance équilibrée continue d'être le centre gravitationnel stable de l'économie (l'attracteur unique). Dans un tel contexte, il n'y a aucune raison d'écarter l'idée que les agents devraient pouvoir connaître les résultats de leurs actions à moyen et long terme, et se comporter de manière à éviter les situations non optimales. Des hypothèses raisonnables de formation des anticipations 
devraient, en particulier, entraîner la disparition des turbulences temporaires, telles que l'effet-machine.

En fait, l'analyse de Hicks est une pièce importante de l'analyse des dynamiques transitoires, mais elle a ses limites. Elle doit être complétée en prenant, explicitement, en considération les problèmes d'information et les difficultés de coordination qui leur sont liés.

Les problèmes de coordination viennent de l'imparfaite articulation entre le fonctionnement du côté production de l'économie et le fonctionnement des marchés, qui empêche d'avoir une croissance auto-entretenue. Pour pouvoir les analyser, il faut mettre l'accent sur l'interaction entre le court et le long terme, dans le cadre d'un modèle qui associe la dimension séquentielle de la production à celle des décisions.

\section{UN MODÈLE NÉO-AUTRICHIEN}

La référence à un modèle néo-autrichien permet de mettre en évidence la dimension temporelle de la production, dont nous venons de souligner l'importance pour une analyse du changement hors de l'équilibre.

Considérons une économie très simple où un produit final homogène est obtenu à partir d'un input primaire (le travail) au moyen d'un processus de production prenant place à travers une séquence de périodes finies $0,1,2, \ldots, n$, $n+1, \ldots, n+\mathrm{N}$, qui comprend une phase de construction $c$ (de la période 0 à la période $n$ ) et, à la suite de celle-ci, une phase d'utilisation $u$ (de la période $n+1$ à la période $n+\mathrm{N}$ ) de la capacité productive. Le processus élémentaire de production est, alors, défini par les vecteurs :

$$
\begin{gathered}
a^{c}=\left[a_{0}, a_{1}, a_{\mathrm{n}}\right] \\
a^{u}=\left[a_{n+1}, \ldots, a_{n+\mathrm{N}}\right] \\
b=\left[b_{n+1}, \ldots, b_{n+\mathrm{N}}\right]
\end{gathered}
$$

dont les éléments représentent, respectivement, les quantités de travail requises par le processus dans les différentes périodes de la phase de construction et de la phase d'utilisation, et les quantités de produit final obtenues dans les différentes périodes de la phase d'utilisation.

Soit :

$$
\begin{gathered}
x^{\mathcal{c}}(t)=\left[x_{o}(t), \ldots, x_{n}(t)\right] \\
x^{u}(t)=\left[x_{n+1}(\mathrm{t}), \ldots, x_{n+\mathrm{N}}(t)\right]
\end{gathered}
$$

les vecteurs des processus, respectivement, encore en phase de construction ou déjà en phase d'utilisation, de telle sorte que la structure de la capacité productive à chaque moment du temps $t$ est représentée par le vecteur :

$$
x(t)=\left[x^{c}(t), x^{u}(t)\right]
$$

dont chaque élément représente un nombre de processus de production d'un âge particulier. 
Par ailleurs, comme effet du vieillissement et du déclassement, le nombre réel de processus de production mis en œuvre à chaque moment $t$ est tel que :

$$
\begin{aligned}
& x_{j}(t)=\tilde{x}_{j}(t)-u_{j}(t) j=1, \ldots, n+\mathrm{N} \\
& \tilde{x}_{j}(t)=x_{j-1}(t-1) ; \tilde{x}_{o}(t)=u_{o}(t)
\end{aligned}
$$

où $u_{o}(t)$ est le taux de natalité des nouveaux processus et $u_{j}(t)$ le nombre des processus qui ont été déclassés.

$u_{o}, x^{c}$ et $x^{u}$ sont déterminés à chaque moment comme solutions du modèle. En particulier, étant donné les contraintes de ressources et les règles de déclassement, les décisions courantes de production déterminent $x^{u}$ et les décisions de construction (d'investissement) déterminent $x^{c}$ and $u_{o}$. Le déclassement de processus de production $\left(u_{j}(t)\right)$ se produit quand les contraintes de ressources humaines ou financières sont si fortes qu'elles ne permettent pas de mettre en œuvre la totalité des processus hérités du passé. Alternativement, une simple diminution du taux d'utilisation de la capacité pourrait être envisagée, moyennant, néanmoins, un coût en travail pour en assurer la maintenance.

Deux classes d'agents économiques sont considérées : les firmes qui utilisent les processus de production pour obtenir un produit final donné, et les ménages. Tous les échanges sont médiatisés par un actif financier qui est ici la monnaie. Les ressources requises pour mettre en œuvre la production et soutenir la consommation, sont, donc, des ressources financières, et non du produit physique (comme c'était le cas dans le modèle néo-autrichien de J.R. Hicks [1973]). Aussi bien pour les producteurs que les consommateurs, leurs ressources viennent de leur participation à l'activité productive. Des ressources financières externes (c'est-à-dire des ressources qui ne viennent pas de l'activité productive : monnaie fiduciaire, endettement bancaire) sont requises quand l'activité productive croît, suivant un taux de croissance positif.

À chaque période, le niveau d'activité des firmes est contraint par les ressources financières disponibles ou, alternativement, par l'offre de travail. Soit $m(t-1)$ le produit des ventes de produit final de la période précédente, $f(t)$ les ressources financières externes, $w(t)$ le fonds des salaires - c'est-à-dire les ressources dévolues à financer le travail employé sur les différents processus de production $-c(t)$ les ressources dévolues à la consommation par les producteurs (ou le prélèvement) et, par conséquent, retirées du financement de la production, $h^{f}(t)$ les encaisses oisives des firmes (désirées $h_{d}^{f}(t)$, et non désirées $h_{n d}^{f}(t)$ ). Alors, la contrainte de financement des firmes, qui détermine $w(t)$, peut être écrite :

$$
m(t-1)+h^{f}(t-1)+f(t)=w(t)+c(t)+h_{d}^{f}(t)
$$

$\operatorname{avec} w(t) \leqslant \hat{w}(t)$

ou alternativement :

$$
m(t-1)+h^{f}(t-1)+f(t)=\hat{w}(t)+c(t)+h_{d}^{f}(t)+h_{n d}^{f}(t)
$$

$\operatorname{avec} w(t)>\hat{w}(t)$

où $\hat{w}(t)$ est le fonds des salaires contraint par la ressource humaine disponible, étant donné le taux de salaire courant $\omega(t)$, soit $\hat{w}(t)=\omega(t) l^{s}(t)$ si $l^{s}(t)<$ $l^{d}(t)$ où $l^{d}(t)$ et $l^{s}(t)$ sont, respectivement, la demande et l'offre de travail.

Quand $w(t)>\hat{w}(t)$, c'est-à-dire quand les firmes sont contraintes dans leur activité par les ressources humaines, le manque de travail établit une contrainte 
sur les décisions d'investissement, qui se traduit par une diminution du taux de natalité, voire par le déclassement de processus en cours de construction. Les ressources financières disponibles, non utilisées, se transforment en encaisses oisives non désirées, qui sont le signe de l'existence d'un déséquilibre :

$$
h_{n d}^{f}(t)=w(t)-\hat{w}(t)
$$

Nous supposons que les producteurs ne veulent pas conserver ces encaisses et, par suite, essaient de les utiliser à partir de la période suivante.

Dans le modèle $h_{d}^{f}(t), f(t)$ et $c(t)$ sont expliqués, soit par des fonctions exogènes (et sont, de fait, des paramètres), soit par des mécanismes endogènes.

Les dépenses de consommation des ménages dépendent aussi des ressources financières disponibles. Soit $y(t)$ la valeur monétaire de la demande courante de produit final et $h^{h}(t)$ les encaisses des ménages (désirées $h_{d}^{h}(t)$ et non désirées $\left.h_{n d}^{h}(t)\right)$. Alors, la contrainte de financement des ménages peut être écrite :

$$
y(t)=w(t)+c(t)+h^{h}(t-1)-h_{d}^{h}(t)
$$

Sous l'hypothèse (qui sera retenue dans ce qui suit) où tous les salaires sont dépensés en biens de consommation, $h_{d}^{h}(t)=0$.

Les prix et les salaires sont fixés au début de chaque période et ne peuvent pas changer durant la période; des changements peuvent seulement prendre place à la jonction de deux périodes. Cela implique que le produit monétaire des ventes des firmes est donné par :

$$
m(t-1)=\min [p(t-1) s(t-1) ; p(t-1) d(t-1)]
$$

où $d$ est la demande courante de produit final, $s$ l'offre courante (qui inclut des stocks non désirés remis sur le marché), et $p$ son prix.

Des variations de stocks de produit final ou de monnaie sont donc un substitut aux changements de prix, à l'intérieur de chaque période. Une offre excédentaire se traduit par une accumulation non désirée de stocks de produit par les firmes :

$$
o(t)=s(t)-d(t)>0
$$

alors que l'excès de demande se traduit par une accumulation non désirée d'encaisses oisives par les ménages :

$$
h_{n d^{\prime}}^{h}(t)=p(t)[d(t)-s(t)]>0
$$

Les ménages ne veulent pas garder ces encaisses oisives et essaient de les utiliser pour l'achat de biens de consommation à la période suivante.

Les changements de prix et de salaires, qui prennent place entre les périodes, sont donnés par :

$$
g_{p}(t)=\kappa \xi(t-1)
$$

où $g_{p}(t)$ est le taux de variation du prix, et

$$
\xi(t-1)=[d(t-1)-s(t-1)] / s(t-1)
$$

le taux de demande excédentaire de produit final ; et par :

$$
g_{\omega}(t)=v \phi(t-1)
$$

où

$$
\phi(t-1)=\left[l^{d}(t-1)-l^{s}(t-1)\right] / l^{s}(t-1)
$$


est le taux de demande excédentaire de travail. $\kappa$ et $v$ sont des coefficients de réaction donnés. Différentes valeurs de ces coefficients correspondent à différents mécanismes de régulation censés eux-mêmes représenter des organisations de marché alternatives. Ainsi, alors que $\kappa, v=1$ expriment une détermination des prix dans le but de solder les marchés, des valeurs plus petites que l'unité de ces coefficients représentent des prix et des salaires institutionnellement déterminés dans le sens où elles renvoient à l'existence d'intermédiaires sur les marchés (marchands, syndicats).

La demande de produit final, en volume, à chaque période, est donnée par :

$$
d(t)=y(t) / p(t)
$$

L'offre de produit final, en volume, à chaque période, est donnée par :

$$
s(t)=\left[m^{*}(t)\right] / p(t)
$$

où

$$
m^{*}(t)=m(t-1)\left\{1+g_{m}(t-1)\right\}
$$

est la valeur monétaire de la demande finale anticipée par les firmes.

La production courante sera alors :

$$
q(t)=s(t)-\eta o(t-1), o<\eta<1
$$

où $\eta$ représente la fraction des stocks réels existant remis sur le marché. En outre :

$$
q(t) \leqslant b_{n} \tilde{x}_{n}(t)+\ldots+b_{n+\mathrm{N}} \tilde{x}_{n+\mathrm{N}}(t)
$$

Le vecteur des processus de production en cours d'utilisation $x^{u}(t)$ est alors déterminé, compte tenu de la règle de déclassement, et la fraction du fonds des salaires requise pour financer la mise en œuvre de ces processus est donnée par :

$$
w^{u}(t)=\omega(t) a^{u} x^{u^{\prime}}(t)
$$

Les ressources disponibles pour financer les processus en construction sera alors :

$$
i(t)=w(t)-w^{u}(t)
$$

Elles déterminent, compte tenu de la règle de déclassement, le vecteur des processus de production en cours de construction.

Le déclassement des processus dépend de la magnitude de la demande finale, s'agissant des processus en cours de construction, et de la disponibilité des ressources financières ou humaines, s'agissant des processus en cours d'utilisation. Quand il y a une contrainte de demande finale, les premiers processus en cours d'utilisation à être déclassés sont les plus anciens ; quand il y a une contrainte financière ou de ressources humaines, les premiers processus en cours de construction à être déclassés sont les plus jeunes. Ces règles de déclassement expriment un critère de flexibilité.

Enfin l'emploi total, à la période $t$, sera donné par :

$$
\mathrm{E}(t)=a^{u} x^{u^{\prime}}(t)+a^{c} x^{c^{\prime}}(t)
$$




\section{LEÇONS À PARTIR D'EXERCICES DE SIMULATION DE DYNAMIQUES TRANSITOIRES}

À l'aide du modèle qui vient d'être présenté, nous entendons analyser les conditions dans lesquelles une accélération de la croissance de l'économie peut être obtenue, dans le but de réduire un taux de chômage pré-existant.

Étant donné la relation inverse qui existe, à l'équilibre, entre la part du prélèvement (ou consommation à partir des profits), $\mu$, et le taux de croissance, $g$, une telle accélération devrait pouvoir être obtenue au moyen d'une diminution de $\mu$, qui s'analyse, en l'occurrence, comme une augmentation du taux d'épargne des entreprises.

Nous partons d'une économie en régime régulier dans laquelle une croissance démographique, qui devient supérieure à la croissance du produit, entraîne une augmentation du taux de chômage et une baisse corrélative du taux de salaire.

Une diminution instantanée et définitive du taux de prélèvement, $\mu$, toutes choses égales par ailleurs, et notamment en laissant le montant de ressources monétaires externes évoluer suivant le taux de croissance courant de l'économie, place l'économie sur un sentier non viable (fig. 1). Une distorsion croissante de la structure de la capacité productive, au bénéfice des processus en cours de construction, va de pair avec un déclassement exponentiel de processus en cours d'utilisation (fig. 2 et 3 ). Il y a trois raisons à cela. La première est que la diminution de $\mu$ en une seule étape engendre une structure de la capacité de l'économie qui ne correspond pas à la structure d'équilibre, puisque, initialement, tout le supplément d'épargne est reporté sur le seul taux de natalité. La deuxième raison est que, étant donné ce qu'est la séquence des paiements, une augmentation du taux de croissance de l'offre de ressources financières externes est requise pour obtenir une augmentation du taux de croissance de l'économie. La troisième raison est qu'en dépit d'un chômage pré-existant le choc est brutal que l'économie est néanmoins confrontée à une contrainte de ressources humaines.

Une diminution progressive du taux de prélèvement, $\mu$, calculée pour mettre en place une structure de la capacité productive compatible avec un taux de croissance de l'économie plus élevé que le taux initial a pour effet, toutes choses égales par ailleurs (donc, en maintenant le taux de croissance des ressources financières externes au niveau de celui du produit), d'engendrer des fluctuations qui sont, progressivement, amorties de telle sorte que l'économie revient sur son sentier initial (fig. 4), mais avec pour corollaire un déclassement exponentiel de processus en cours d'utilisation qui traduit l'inadéquation de la structure de la capacité productive aux conditions générales de la croissance (fig. 5 et 6 ). En fait, l'absence d'une demande supplémentaire explique cette situation. Il suffit, en effet, d'introduire une telle demande - c'est-à-dire des exportations génératrices de ressources financières externes - pour obtenir l'accélération recherchée de la croissance (fig. 7). Ce résultat conforte une intuition de T.R. Malthus pour qui il n'était pas possible d'augmenter le taux de croissance en augmentant simplement le taux d'épargne (et le taux d'investissement).

Une diminution progressive du taux de prélèvement, $\mu$, accompagnée d'une augmentation instantanée et définitive de l'offre de monnaie ne permet pas non plus une accélération de la croissance. Des fluctuations aboutissent à un état non 


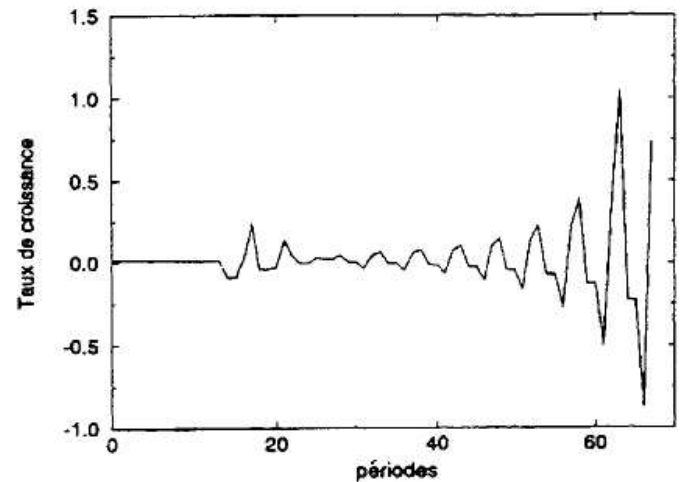

Figure 1.

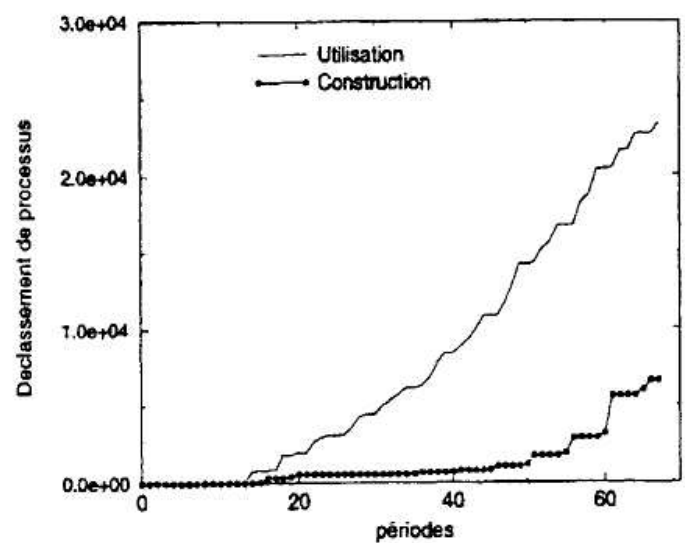

Figure 3.

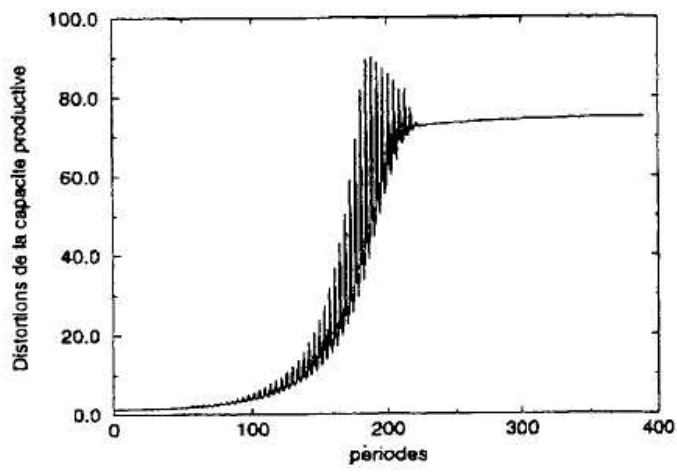

Figure 5.

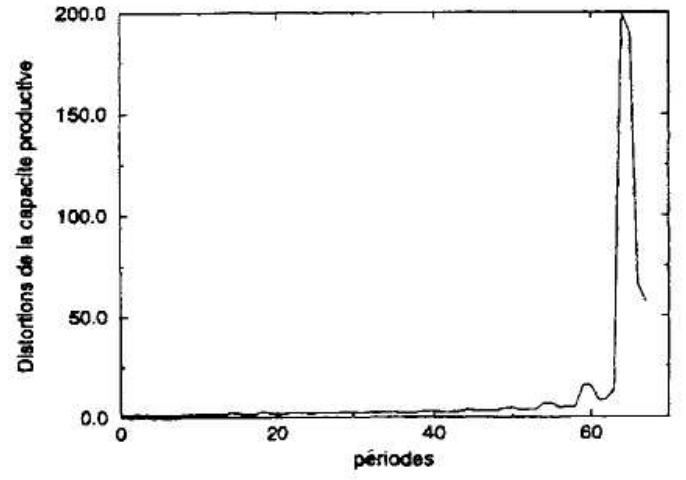

Figure 2.

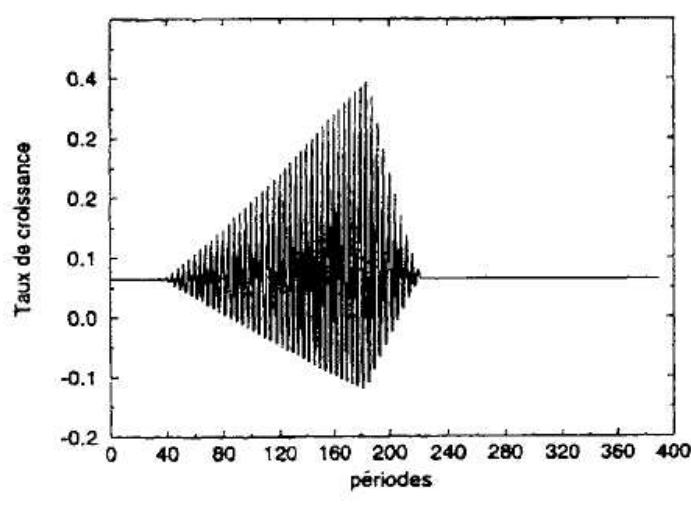

Figure 4.

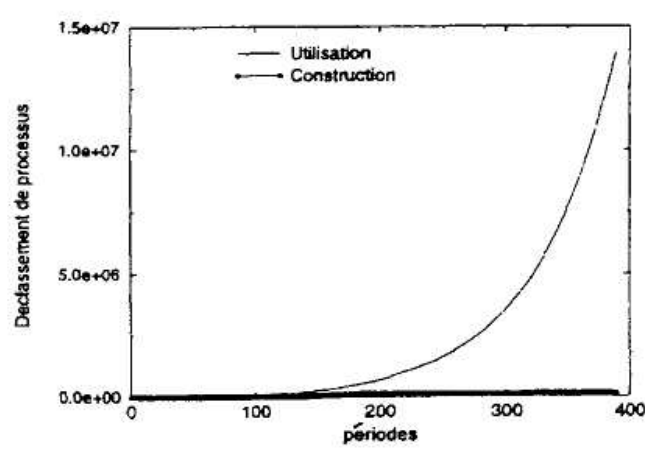

Figure 6. 


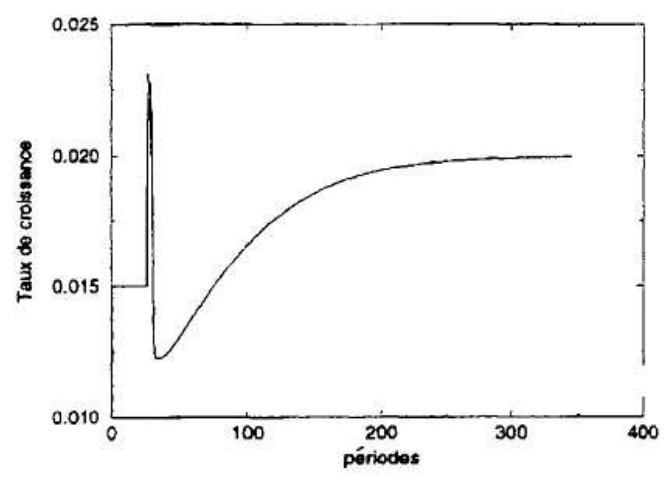

Figure 7.

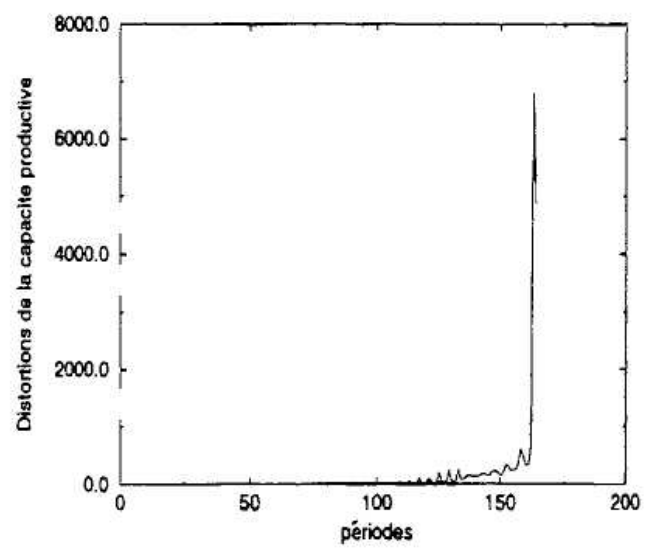

Figure 9.

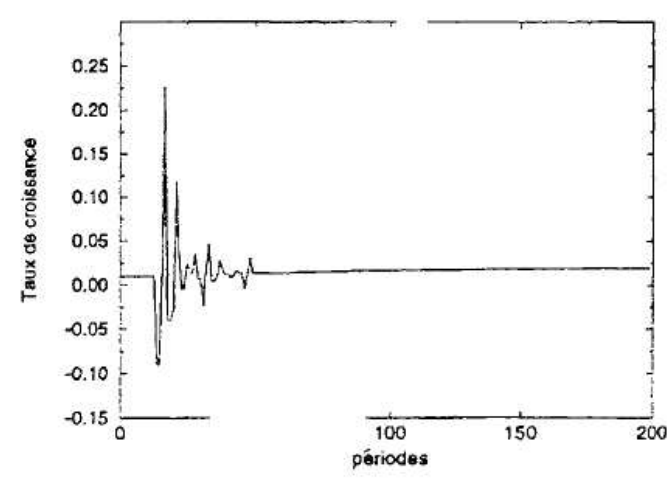

Figure 11.

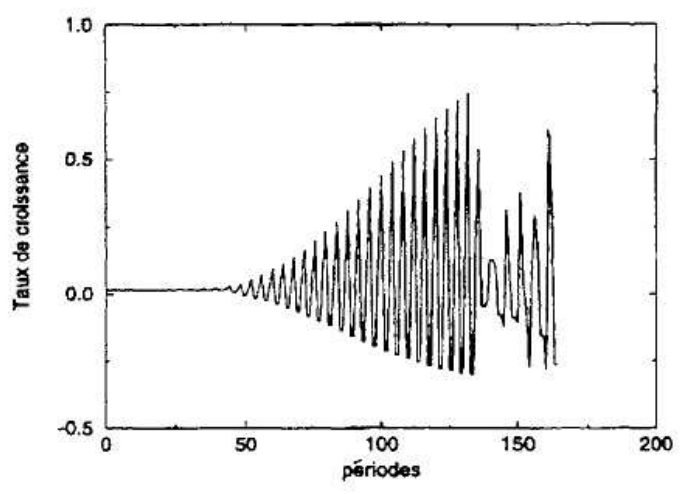

Figure 8.

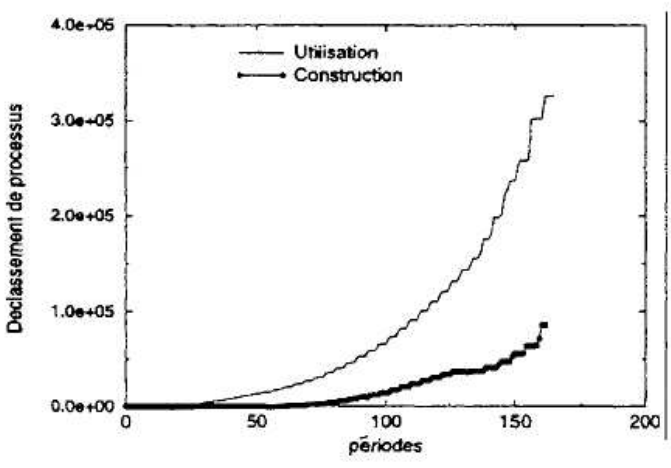

Figure 10.

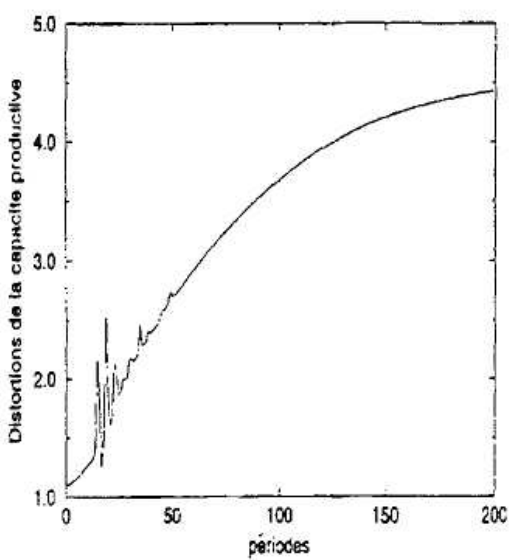

Figure 12. 


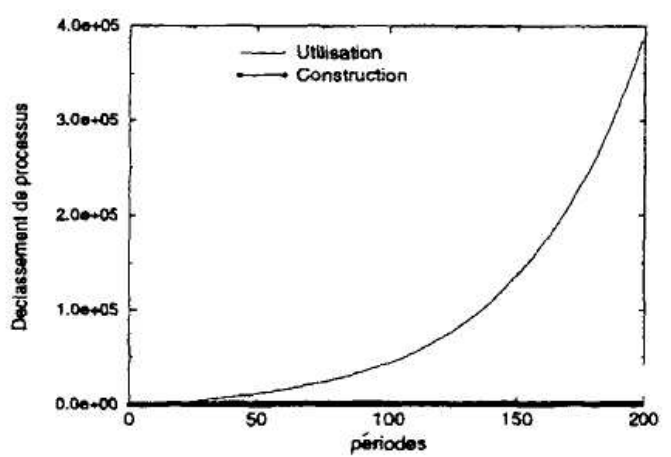

Figure 13.

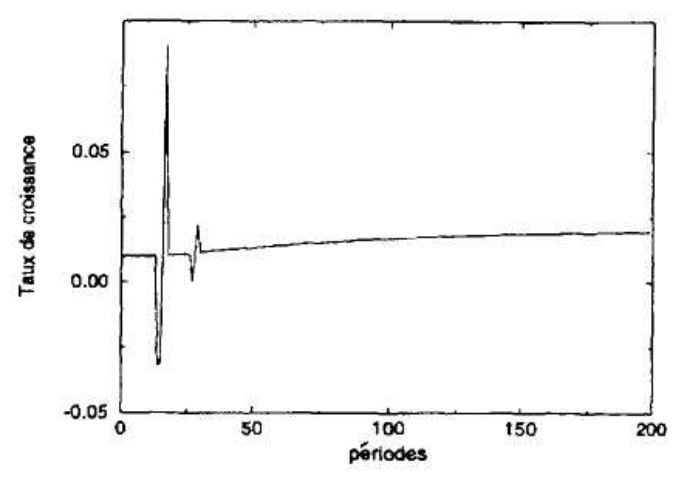

Figure 15.

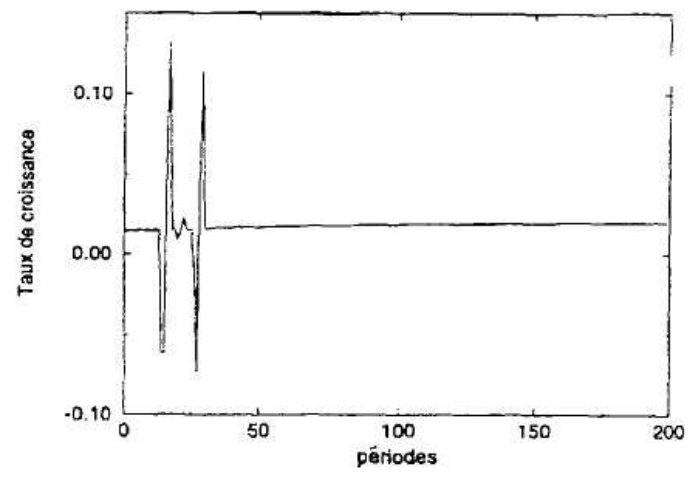

Figure 17.

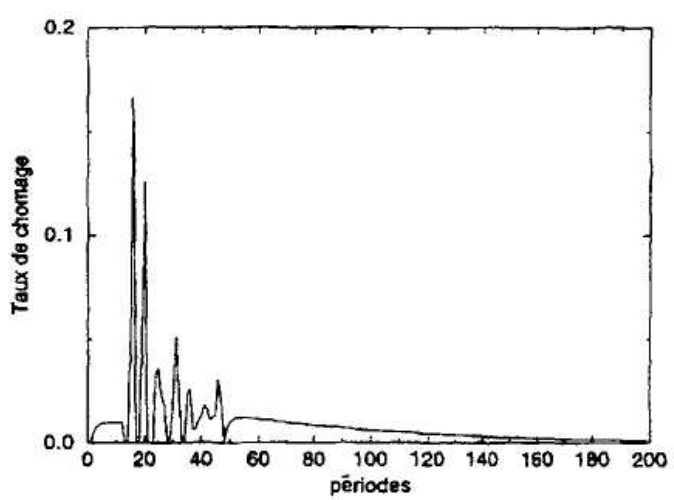

Figure 14.

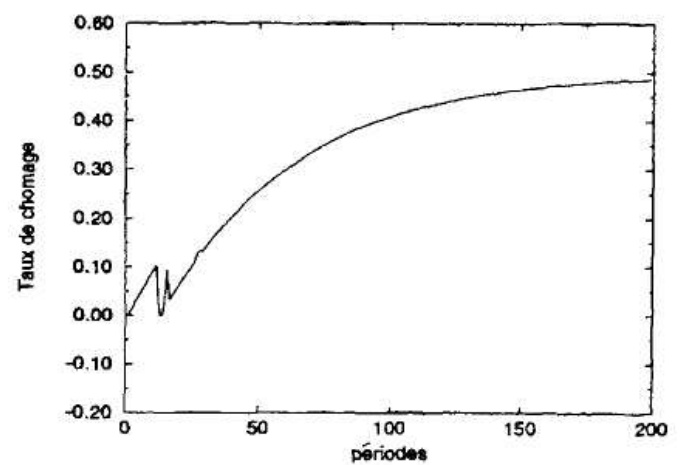

Figure 16.

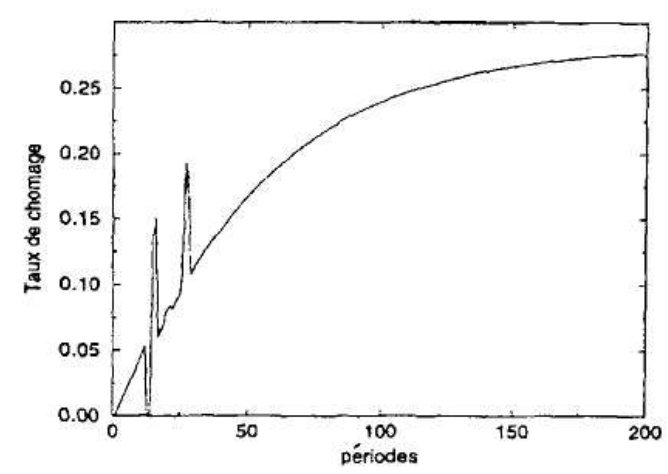

Figure 18. 
viable de l'économie (fig. 8). Cela provient, à l'évidence, d'une distorsion croissante de la structure de la capacité productive (fig. 9) avec pour effet un déclassement exponentiel de processus en cours d'utilisation (fig. 10). Cela est le résultat d'une croissance des ressources monétaires externes disponibles incompatible avec l'évolution du taux de prélèvement (c'est-à-dire du taux d'épargne).

En revanche, une diminution instantanée et définitive du taux de prélèvement couplée avec une augmentation du taux de croissance des ressources monétaires externes peut se traduire par une accélération de la croissance. Dans certains cas précis, en effet, la compatibilité des deux mouvements fait qu'au terme de fluctuations progressivement amorties, l'économie rejoint un sentier de croissance régulière caractérisé par un taux plus élevé que le taux initial (fig. 11), correspondant à une structure de la capacité productive caractérisée par une part plus grande des processus en cours de construction (fig. 12) et le déclassement de processus en cours d'utilisation en excès (fig. 13), et impliquant un retour au plein emploi (fig. 14).

La viabilité dépend, de manière cruciale, de quatre éléments qui interagissent : la part du prélèvement dans le produit, l'intensité de la contrainte de ressources humaines, le niveau de l'apport de ressources financières externes et le mécanisme d'ajustement des salaires. Ainsi, avec les mêmes données que dans le cas précédent, mais en introduisant une rigidité des salaires nominaux à la baisse, on obtient toujours une accélération de la croissance avec de moindres fluctuations dans la phase transitoire (fig. 15) mais avec pour corollaire une augmentation du taux de chômage qui tend vers une valeur asymptotique (fig. 16). $\mathrm{Si}$, maintenant, on considère une situation caractérisée par une contrainte de ressources humaines plus forte - les ressources humaines disponibles sont nettement insuffisantes pour faire face aux besoins de construction de nouveaux processus malgré le chômage existant initialement - alors, l'introduction d'une rigidité des salaires nominaux à la baisse garantit la viabilité et l'accélération de la croissance (fig. 17) et l'augmentation asymptotique du taux de chômage (fig. 18) est moindre que lorsque la contrainte de ressources humaines était plus faible. Ces résultats (qui n'ont d'autre valeur qu'heuristique) illustrent la difficulté d'obtenir une croissance plus forte dans un contexte où existent, simultanément, des complémentarités intertemporelles et des difficultés de coordination.

Ce sont, d'une manière générale, les conditions dans lesquelles s'ajustent les prix et les salaires en réaction aux déséquilibres de marché, qui témoignent des difficultés de coordination et qui caractérisent le mécanisme d'acquisition d'information. Couplées aux distorsions de la capacité productive, elles sont au cœur de la dynamique transitoire.

\section{CONCLUSION}

Dans un modèle où la dimension temporelle de la production est véritablement prise en considération, une simple perturbation, qui rompt l'équilibre intertemporel entre les processus de production, suscite des mécanismes d'évolution éventuellement complexes, pouvant mettre en péril la viabilité de l'éco- 
nomie. L'effet combiné des ajustements aux déséquilibres de marché et des ajustements de la structure de la capacité productive rend difficile d'atteindre un objectif global tel qu'une accélération de la croissance, par ailleurs jugée utile pour, par exemple, résorber le chômage et jugée crédible sur la base de la seule comparaison des régimes réguliers. Cela démontre qu'il est important, dans la perspective d'établir des règles de politique économique, de s'interroger sur les mécanismes de la croissance hors de l'équilibre, plutôt que sur les sources ou les facteurs d'une croissance équilibrée.

\section{REFERENCES BIBLIOGRAPHIQUES}

BarRo R.J., SAla Y., MARTIN X. [1995], Economic Growth, New York, McGraw Hill.

GEORGESCU-RoEGEN N. [1976], "Dynamics Models and Economic Growth », dans Energy and Economic Myths, Oxford, Pergamon Press.

HICKS J.R. [1973], Capital and Time, Oxford, Clarendon Press.

Solow R.M. [1992], Siena Lectures on Endogenous Growth, ed. by Serena Sordi, Universita degli studi di Siena, Dipartemento di Economia Politica.

Solow R.M. [1994], « Perspectives on Growth Theory », Journal of Economic Perspectives, 8, p. 45-55. 